MSMPathfinder: Identification of pathways in Markov state models Supplementary Information

Daniel Nagel, Anna Weber and Gerhard Stock a

Biomolecular Dynamics, Institute of Physics, Albert Ludwigs University, 79104 Freiburg, Germany.

(Dated: 2 November 2020)

\title{
I. METHODS
}

\section{I.1. Other definitions of cutting loops}

In the main paper we motivated the need for cutting unproductive loops to analyze pathways. This cutting can be defined arbitrarily different. In the following we briefly discuss three different ways. One can either cut at occurrence first

$$
1 \rightarrow \underbrace{2 \rightarrow 5 \rightarrow 3 \rightarrow 4 \rightarrow 2}_{\text {cutting occurring first }} \rightarrow 3 \rightarrow 6 \rightarrow 7 \rightarrow 4 \Rightarrow 1 \rightarrow 2 \rightarrow 3 \rightarrow 6 \rightarrow 7 \rightarrow 4
$$

or cut the longest loop first

$$
1 \rightarrow 2 \rightarrow 5 \rightarrow 3 \rightarrow \underbrace{4 \rightarrow 2 \rightarrow 3 \rightarrow 6 \rightarrow 7 \rightarrow 4}_{\text {cutting longest first }} \Rightarrow 1 \rightarrow 2 \rightarrow 5 \rightarrow 3 \rightarrow 4
$$

or finally cut the shortest loop first

$$
1 \rightarrow 2 \rightarrow 5 \rightarrow \underbrace{3 \rightarrow 4 \rightarrow 2 \rightarrow 3}_{\text {cutting shortest first }} \rightarrow 6 \rightarrow 7 \rightarrow 4 \Rightarrow 1 \rightarrow 2 \rightarrow 5 \rightarrow 3 \rightarrow 6 \rightarrow 7 \rightarrow 4
$$

a) e-mail:stock@physik.uni-freiburg.de 


\section{I.2. Removing self-transition rates}

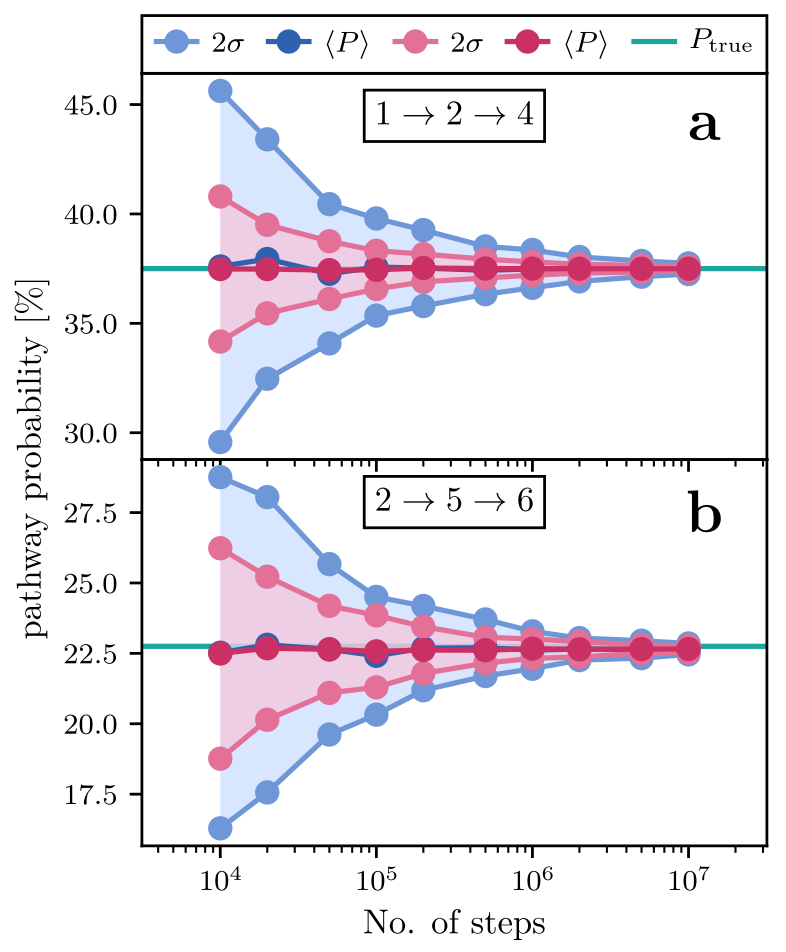

FIG. S1: Convergence of an MCMC simulation for the pathway probability $P$ of major pathway (a) $1 \rightarrow 2 \rightarrow 4$ (4-state model, see Fig. 1) and (b) $2 \rightarrow 5 \rightarrow 6$ (6-state model, see Fig. S3). Showing mean (blue) and standard error (light blue) of an MCMC run, mean (red) and standard error (light red) of an MCMC with removed self-transition rates [Eq. (2)] and theoretical value $P_{\text {true }}$ (green).

\section{I.3. Implementation and convergence of MSMPathfinder}

The $\mathrm{C}++$ version of MSMPathfinder provided at github.com/moldyn/MSMPathfinder contains an implementation of MCMC and the MSMPathfinder method presented in the main paper. Dealing with relatively small graphs, we process the sampled pathways on-thefly, storing not finished ones in the RAM. Therefore, only the part of the MCMC simulation for the currently not yet completed pathway needs to be stored. The program processes approx. $40 \times 10^{6}$ steps/s on a single core of an Intel Xeon E-2134 CPU.

In the following we compare the wall-clock time for varying $N$ states and $k$ edges. The transistion matrices $T$ are build by overlapping submatrices of size $k \times k$ were the diagonal is metastable $\left(T_{i i} \approx 1\right)$, with additional off-diagonal edges to get short and long pathways. An example for $k=3$ and $N=5,7,9$ is

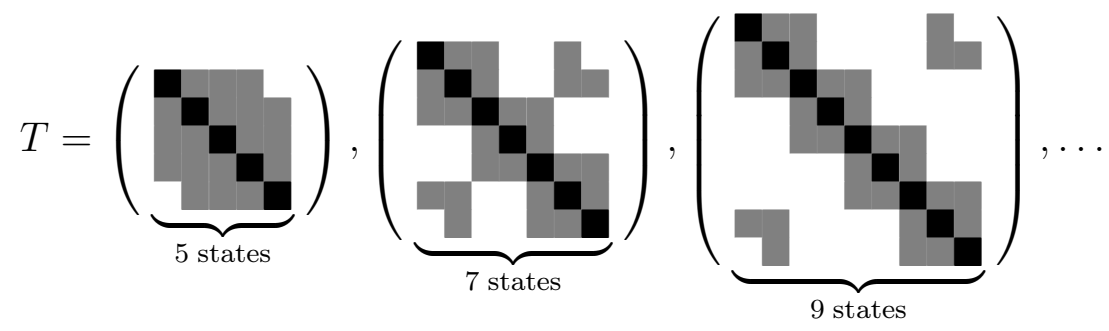


where black squares correspond to values close to one, gray squares to edges with low probability and white squares to edges with zero probability. For each combination of edges and states, 50 independent matrices were used to check the wall-clock time needed to converge to a total missing pathspace of less than $\varepsilon$. The results are visualized in Fig. S2, Often, the path probability density is localized, which improves the convergence. ${ }^{1}$ The 22 states model of $\mathrm{AIB}_{9}$ used in Table $\mathrm{S} 3$, containing only two vanishing transition probabilities, took 813 s to converge to $\varepsilon=0.56 \%$ on a single core of an Intel Xeon E-2134 CPU.

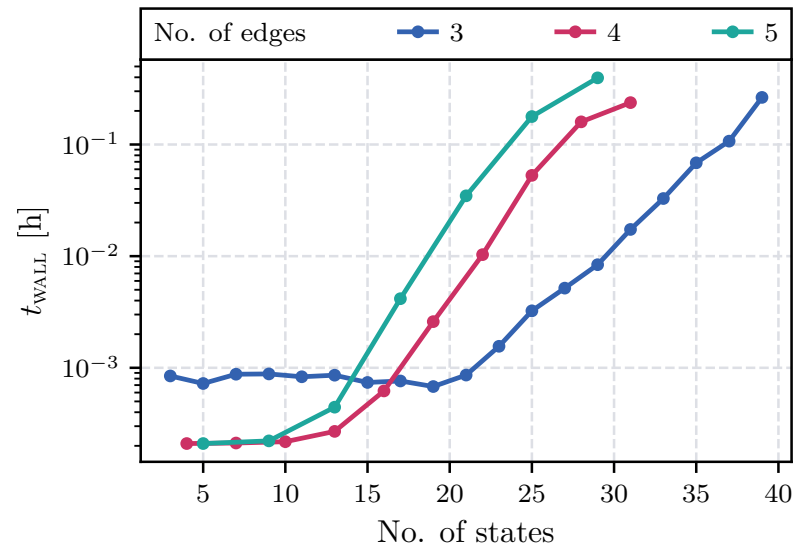

(a) $\varepsilon=1 \%$

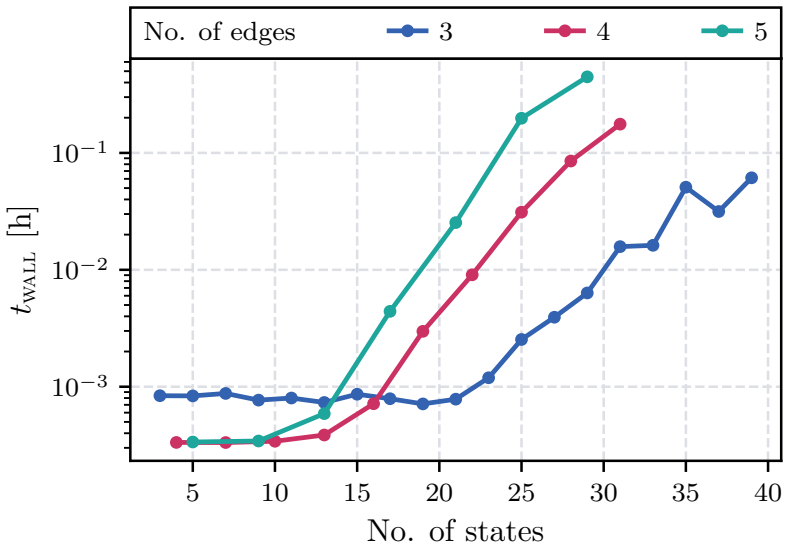

(b) $\varepsilon=5 \%$

FIG. S2: The time wall-clock time $t_{\text {waLL }}$ needed for converging to a total error $\varepsilon$ of less than (a) one percent and (b) five percent. As reference a single core of an Intel Xeon E-2134 CPU was used.

\section{RESULTS}

\section{II.1. Few-state models}

\section{4-state model}

Symmetric transition matrix of the 4-state model shown in Fig. 1,

$$
T=\left(\begin{array}{llll}
0.92 & 0.04 & 0.04 & 0.00 \\
0.10 & 0.70 & 0.10 & 0.10 \\
0.10 & 0.10 & 0.70 & 0.10 \\
0.00 & 0.04 & 0.04 & 0.92
\end{array}\right)
$$




\section{6-state model}

$\mathbf{a} T=\left(\begin{array}{llllll}0.90 & 0.03 & 0.07 & 0.00 & 0.00 & 0.00 \\ 0.05 & 0.63 & 0.15 & 0.07 & 0.10 & 0.00 \\ 0.08 & 0.04 & 0.60 & 0.14 & 0.14 & 0.00 \\ 0.00 & 0.12 & 0.15 & 0.50 & 0.15 & 0.08 \\ 0.00 & 0.04 & 0.16 & 0.10 & 0.60 & 0.10 \\ 0.00 & 0.00 & 0.00 & 0.08 & 0.02 & 0.90\end{array}\right)$

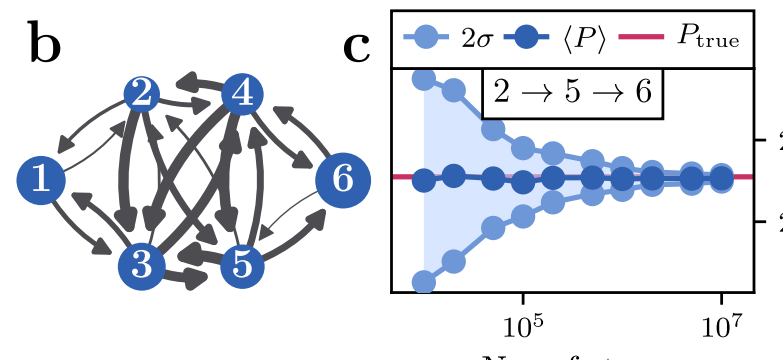

No. of steps d

\begin{tabular}{lrr}
\hline \multirow{2}{*}{ pathways $1,2 \rightarrow 6$} & \multicolumn{2}{c}{ pathway probability [\%] } \\
& MCMC & TPT \\
\hline $2 \rightarrow 5 \rightarrow 6$ & 22.6 & 29.0 \\
$2 \rightarrow 3 \rightarrow 5 \rightarrow 6$ & 13.3 & 0.7 \\
$1 \rightarrow 3 \rightarrow 5 \rightarrow 6$ & 12.5 & 26.2 \\
$2 \rightarrow 4 \rightarrow 6$ & 9.7 & 9.5 \\
$2 \rightarrow 3 \rightarrow 4 \rightarrow 6$ & 8.5 & 27.2 \\
$1 \rightarrow 3 \rightarrow 4 \rightarrow 6$ & 8.0 & - \\
$2 \rightarrow 4 \rightarrow 5 \rightarrow 6$ & 5.3 & 7.5 \\
$2 \rightarrow 5 \rightarrow 4 \rightarrow 6$ & 4.0 & - \\
$2 \rightarrow 3 \rightarrow 4 \rightarrow 5 \rightarrow 6$ & 4.0 & - \\
$1 \rightarrow 3 \rightarrow 4 \rightarrow 5 \rightarrow 6$ & 3.7 & - \\
$2 \rightarrow 5 \rightarrow 3 \rightarrow 4 \rightarrow 6$ & 2.3 & - \\
$2 \rightarrow 3 \rightarrow 5 \rightarrow 4 \rightarrow 6$ & 2.1 & - \\
$1 \rightarrow 3 \rightarrow 5 \rightarrow 4 \rightarrow 6$ & 2.0 & - \\
$2 \rightarrow 4 \rightarrow 3 \rightarrow 5 \rightarrow 6$ & 1.9 & - \\
\hline
\end{tabular}

FIG. S3: (a) Transition matrix of an asymmetric 6-state model. (b) Visualization of 6-state model, where the node sizes correspond to the stationary populations $\pi_{i}$ and the edge weight to the transition probabilities $T_{i j}$. (c) Convergence of an MCMC simulation for the pathway probability $P$ of major pathway $2 \rightarrow 5 \rightarrow 6$, showing mean (blue), standard error (light blue) and theoretical value $P_{\text {true }}(\mathrm{red})$. (d) List of the pathways between $1,2 \rightarrow 6$ obtained by MCMC and TPT including their pathway probabilities. 


\section{II.2. Peptide helix AIB $_{9}$}

\section{States definition}

TABLE S1: List of populations and conformational structures for all microstates of the 46-state model of $\mathrm{AIB}_{9}$ generated by Perez et al. with $l$ and $r$ standing for the left and right-handed helical conformation, while $l^{*}$ and $r^{*}$ correspond to the excited conformations..$^{2}$

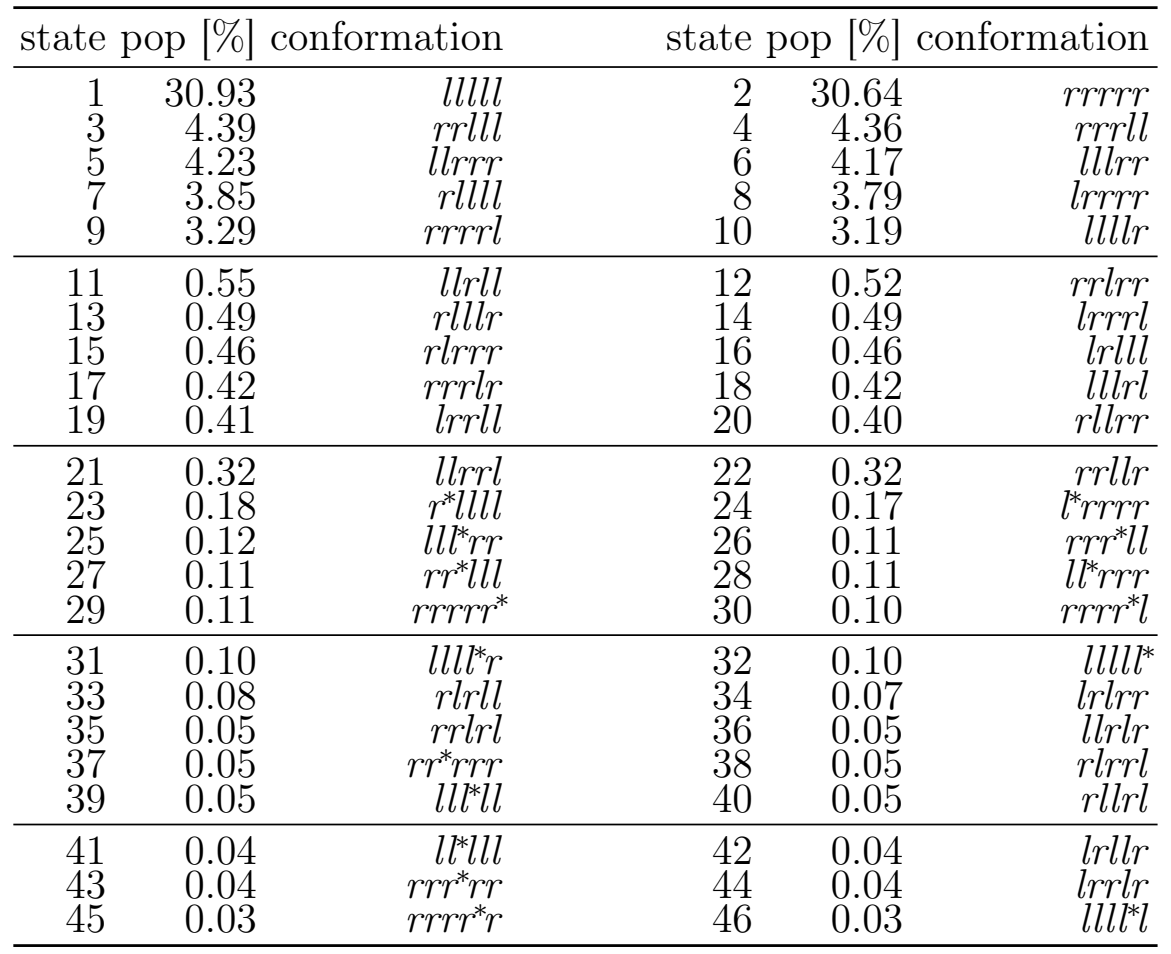

\section{Lumped pathways of full 46-state model}

TABLE S2: Probabilities of lumped pathways going from left-handed lllll to right-handed rrrrr conformation obtained by MSMPathfinder and TPT for full 46-state model of $\mathrm{AIB}_{9}$. Two squares connected by a + mean that pathways involve states associated with the second square and possibly also with the first. Three squares mean that pathways involve green labeled states and also states associated with the other two colors. All excited states are included in the remaining pathways. For MSMPathfinder a probability threshold of $P_{\varepsilon}=$ $10^{-11}$ was used resulting in an absolute error of $\varepsilon=2.14 \%$.

\begin{tabular}{|c|c|}
\hline states of pathways & 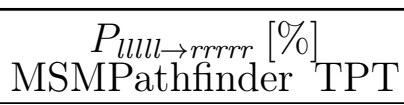 \\
\hline direct pathway & $\begin{array}{rr}1.09 & 1.07\end{array}$ \\
\hline$=$ rllll, rrlll, rrrll, rrrrl & 25.4433 .92 \\
\hline$+\quad=l r l l l$, lrrll, rrllr, rrrlr & $6.65 \quad 6.25$ \\
\hline$=l l l l r$, lllrr, llrrr, lrrrr & 22.7530 .89 \\
\hline$+=l l l r l, l l r r l, r l l r r, r l r r r$ & $6.56 \quad 6.38$ \\
\hline$=l l r l l$, rlllr, lrrrl, $r r l r r$ & 1.26 \\
\hline & 4.44 \\
\hline & $4.51 \quad 2.65$ \\
\hline remaining pathways & 27.3013 .8 \\
\hline
\end{tabular}




\section{Chiral transition pathways of peptide helix $\mathrm{AIB}_{9}$}

TABLE S3: Pathways of $\mathrm{AIB}_{9}$ going from $l l l l l \rightarrow r r r r$ calculated with MSMPathfinder. A threshold of $P_{\varepsilon}=5 \times 10^{-12}$ was chosen which resulted in an error of $\varepsilon=0.56 \%$. Here the shorthand notations for the states from Tab. S1 are used.

\begin{tabular}{|c|c|c|}
\hline \# pathway & $P[\%]$ & $\sum P[\%] \tau[\mathrm{ns}]$ \\
\hline $11-$ & 2.87 & 2.8739 .74 \\
\hline$\rightarrow 3 \rightarrow 4 \rightarrow 2$ & 2.66 & 5.5441 .30 \\
\hline $31 \rightarrow 6 \rightarrow 5 \rightarrow 2$ & 2.62 & 8.1639 .72 \\
\hline $41 \rightarrow 7 \rightarrow 4 \rightarrow 2$ & 2.58 & 10.7439 .61 \\
\hline $51 \rightarrow 6 \rightarrow 5 \rightarrow 8 \rightarrow 2$ & 2.43 & 13.1741 .29 \\
\hline $61 \rightarrow 6 \rightarrow 8 \rightarrow 2$ & 2.15 & 15.3239 .61 \\
\hline $71 \rightarrow 4 \rightarrow 2$ & 2.07 & 17.3937 .68 \\
\hline $81 \rightarrow 3 \rightarrow 2$ & 2.07 & 19.4637 .82 \\
\hline $91 \rightarrow 3 \rightarrow 4 \rightarrow 9 \rightarrow 2$ & 1.96 & 21.4241 .09 \\
\hline $101 \rightarrow 7 \rightarrow 3 \rightarrow 2$ & 1.94 & 23.3639 .42 \\
\hline $111 \rightarrow 5$ & 1.92 & 25.2737 .83 \\
\hline $121-$ & 1.86 & 27.1437 .68 \\
\hline $131-$ & 1.82 & 28.9639 .38 \\
\hline $3 \rightarrow 4 \rightarrow 9 \rightarrow 2$ & 1.82 & 30.7842 .65 \\
\hline $151-$ & 1.80 & 32.5739 .43 \\
\hline$\rightarrow 6 \rightarrow 5 \rightarrow 2$ & 1.80 & 34.3741 .06 \\
\hline $4 \rightarrow 9 \rightarrow 2$ & 1.77 & 36.1440 .98 \\
\hline $181 \rightarrow$ & 1.73 & 37.8739 .38 \\
\hline $7 \rightarrow 3 \rightarrow 9 \rightarrow 2$ & 1.70 & 39.5740 .97 \\
\hline $10 \rightarrow 6 \rightarrow 5 \rightarrow 8 \rightarrow 2$ & 1.67 & 41.2442 .63 \\
\hline $5 \rightarrow 8 \rightarrow 2$ & 1.62 & 42.8640 .98 \\
\hline $221 \rightarrow 10 \rightarrow 6 \rightarrow 8 \rightarrow 2$ & 1.48 & 44.3440 .97 \\
\hline $23 \stackrel{1 \rightarrow 4 \rightarrow 9 \rightarrow 2}{\rightarrow}$ & 1.42 & 45.7739 .06 \\
\hline $241 \rightarrow 10 \rightarrow 6 \rightarrow 2$ & 1.29 & 47.0539 .05 \\
\hline $251 \rightarrow 7 \rightarrow 2$ & 1.20 & 48.2537 .19 \\
\hline $261 \rightarrow 2$ & 1.17 & 49.4134 .85 \\
\hline $271 \rightarrow$ & 1.13 & 50.5437 .18 \\
\hline $28 \quad 1 \rightarrow 7 \rightarrow 9 \rightarrow 2$ & 1.12 & 51.6638 .90 \\
\hline $29 \quad 1 \rightarrow 9 \rightarrow 2$ & 0.89 & 52.5436 .68 \\
\hline $301 \rightarrow 10 \rightarrow 8 \rightarrow 2$ & 0.88 & 53.4238 .91 \\
\hline $311 \rightarrow 10 \rightarrow 2$ & 0.66 & 54.0836 .69 \\
\hline $321 \rightarrow 20 \rightarrow 2$ & 0.42 & 54.5036 .05 \\
\hline $331 \rightarrow 14 \rightarrow 2$ & 0.38 & 54.8836 .12 \\
\hline $341 \rightarrow 13 \rightarrow 2$ & 0.37 & 55.2536 .13 \\
\hline $351 \rightarrow 11 \rightarrow 8 \rightarrow 2$ & 0.32 & 55.5738 .33 \\
\hline $361 \rightarrow 19 \rightarrow 2$ & 0.32 & 55.8936 .07 \\
\hline $371 \rightarrow 16 \rightarrow 4 \rightarrow 2$ & 0.31 & 56.2038 .77 \\
\hline $381 \rightarrow 6 \rightarrow 15 \rightarrow 2$ & 0.31 & 56.5138 .78 \\
\hline $391 \rightarrow 10 \rightarrow 20 \rightarrow 2$ & 0.31 & 56.8237 .87 \\
\hline $401 \rightarrow 3 \rightarrow 17 \rightarrow 2$ & 0.31 & 57.1338 .90 \\
\hline
\end{tabular}


TABLE S4: Pathways of $\mathrm{AIB}_{9}$ going from $l l l l l \rightarrow$ rrrrr calculated with TPT. Here the shorthand notations for the states from Tab. S1 are used.

\begin{tabular}{|c|c|c|}
\hline \# pathv & $P[\%]$ & $\sum P[\%]$ \\
\hline $11 \rightarrow 3 \rightarrow 4$ & 11.24 & 11.24 \\
\hline $21 \rightarrow 6 \rightarrow 5 \rightarrow 2$ & 10.33 & 1.57 \\
\hline $31 \rightarrow 7 \rightarrow 4 \rightarrow 9 \rightarrow 2$ & 6.63 & 8.20 \\
\hline $41 \rightarrow 7 \rightarrow 3 \rightarrow 2$ & .49 & 3.69 \\
\hline $51 \rightarrow 10 \rightarrow 6 \rightarrow 8 \rightarrow 2$ & 5.45 & 39.14 \\
\hline $61 \rightarrow 5 \rightarrow 8 \rightarrow 2$ & 5.14 & 44.28 \\
\hline $71 \rightarrow 7 \rightarrow 3 \rightarrow 9 \rightarrow 2$ & 4.79 & 49.08 \\
\hline $81 \rightarrow 10 \rightarrow 5 \rightarrow 8 \rightarrow 2$ & 4.48 & 53.56 \\
\hline $91 \rightarrow 4 \rightarrow 2$ & 3.65 & 57.21 \\
\hline $101 \rightarrow 6 \rightarrow 2$ & 3.25 & 60.46 \\
\hline $111 \rightarrow 7 \rightarrow$ & 1.72 & .18 \\
\hline $121 \rightarrow 7 \rightarrow 2$ & .57 & .75 \\
\hline $131 \rightarrow 8 \rightarrow 2$ & 1.49 & .24 \\
\hline $141 \rightarrow 10 \rightarrow 8 \rightarrow 2$ & 1.35 & 66.58 \\
\hline $151 \rightarrow 13 \rightarrow 2$ & 1.23 & .82 \\
\hline $161 \rightarrow 14 \rightarrow 2$ & 1.23 & 69.04 \\
\hline $171 \rightarrow 2$ & 1.16 & .21 \\
\hline $181 \rightarrow 9 \rightarrow$ & 1.11 & .31 \\
\hline $191 \rightarrow 4 \rightarrow 9 \rightarrow 2$ & 1.02 & 72.34 \\
\hline $201 \rightarrow 10 \rightarrow 6 \rightarrow 2$ & 1.02 & 73.35 \\
\hline $211 \rightarrow 3 \rightarrow$ & & 74.33 \\
\hline $221 \rightarrow 18 \rightarrow 5 \rightarrow 2$ & 0.88 & 5.21 \\
\hline $231 \rightarrow 20 \rightarrow 2$ & 0.85 & 76.06 \\
\hline $241 \rightarrow 10 \rightarrow 2$ & 0.83 & 76.90 \\
\hline $251 \rightarrow 10 \rightarrow 6 \rightarrow 15 \rightarrow 2$ & 0.82 & 77.72 \\
\hline $261 \rightarrow 19 \rightarrow 4 \rightarrow 9 \rightarrow 2$ & 0.82 & 78.54 \\
\hline $271 \rightarrow 16 \rightarrow 4 \rightarrow 17 \rightarrow 2$ & 0.81 & .35 \\
\hline $281 \rightarrow 10 \rightarrow 6 \rightarrow 20 \rightarrow 2$ & 0.72 & 80.07 \\
\hline $291 \rightarrow 16 \rightarrow 3 \rightarrow 22 \rightarrow 2$ & 0.70 & 80.78 \\
\hline $301 \rightarrow 10 \rightarrow 20 \rightarrow 2$ & 0.70 & 81.48 \\
\hline $311 \rightarrow 19$ & 0.67 & 82.14 \\
\hline $321 \rightarrow 21 \rightarrow 8 \rightarrow 2$ & 0.61 & 82.76 \\
\hline $331 \rightarrow 22 \rightarrow 2$ & 0.57 & 83.32 \\
\hline $341 \rightarrow 21 \rightarrow 5 \rightarrow 15 \rightarrow 2$ & 0.56 & 83.88 \\
\hline $351 \rightarrow 18 \rightarrow 6 \rightarrow 12 \rightarrow 2$ & 0.55 & 84.44 \\
\hline $361 \rightarrow 10 \rightarrow 5 \rightarrow 2$ & 0.55 & 84.98 \\
\hline $371 \rightarrow 19 \rightarrow 9 \rightarrow 2$ & 0.54 & 85.52 \\
\hline $381 \rightarrow 7 \rightarrow 22 \rightarrow 2$ & 0.52 & 86.05 \\
\hline $391 \rightarrow 11 \rightarrow 8 \rightarrow 2$ & 0.52 & 86.57 \\
\hline $401 \rightarrow 21 \rightarrow 2$ & 0.51 & 87.08 \\
\hline
\end{tabular}




\section{State definitions of HP35}

TABLE S5: Statistics for all microstates of the 56-state model of HP35. For more details on the construction we refer to Nagel et al..$^{[3}$

\begin{tabular}{|c|c|c|c|c|c|c|c|}
\hline state & \multicolumn{3}{|c|}{ pop pop [\%] total [\%] } & state & \multicolumn{3}{|c|}{ pop pop [\%] total [\%] } \\
\hline $\begin{array}{l}5 \\
5 \\
7 \\
9\end{array}$ & $\begin{array}{l}327157 \\
24471 \\
87623 \\
64809 \\
48504\end{array}$ & $\begin{array}{r}21.44 \\
8.16 \\
5.74 \\
4.25 \\
3.18\end{array}$ & $\begin{array}{l}21.44 \\
48.05 \\
59.57 \\
69.54 \\
76.08\end{array}$ & $\begin{array}{r}2 \\
4 \\
6 \\
8 \\
10\end{array}$ & $\begin{array}{l}81679 \\
88139 \\
87259 \\
51404 \\
37288\end{array}$ & $\begin{array}{r}18.46 \\
5.78 \\
5.72 \\
3.37 \\
2.44\end{array}$ & $\begin{array}{l}39.90 \\
53.83 \\
65.29 \\
72.90 \\
78.53\end{array}$ \\
\hline $\begin{array}{l}11 \\
13 \\
15 \\
17 \\
19\end{array}$ & $\begin{array}{l}32541 \\
25952 \\
20956 \\
18464 \\
12266\end{array}$ & $\begin{array}{l}2.13 \\
1.70 \\
1.37 \\
1.21 \\
0.80\end{array}$ & $\begin{array}{l}80.66 \\
84.36 \\
87.15 \\
89.71 \\
91.61\end{array}$ & $\begin{array}{l}12 \\
14 \\
16 \\
18 \\
20\end{array}$ & $\begin{array}{l}30569 \\
21525 \\
20701 \\
16754 \\
11939\end{array}$ & $\begin{array}{l}2.00 \\
1.41 \\
1.36 \\
1.10 \\
0.78\end{array}$ & $\begin{array}{l}82.66 \\
85.77 \\
88.50 \\
90.81 \\
92.40\end{array}$ \\
\hline $\begin{array}{l}21 \\
23 \\
25 \\
27 \\
29\end{array}$ & $\begin{array}{r}11559 \\
9158 \\
8152 \\
7370 \\
5221\end{array}$ & $\begin{array}{l}0.76 \\
0.60 \\
0.53 \\
0.48 \\
0.34\end{array}$ & $\begin{array}{l}93.15 \\
94.36 \\
95.49 \\
96.47 \\
97.22\end{array}$ & $\begin{array}{l}22 \\
24 \\
26 \\
28 \\
30\end{array}$ & $\begin{array}{l}9238 \\
9157 \\
7487 \\
6219 \\
4490\end{array}$ & $\begin{array}{l}0.61 \\
0.60 \\
0.49 \\
0.41 \\
0.29\end{array}$ & $\begin{array}{l}93.76 \\
94.96 \\
95.98 \\
96.87 \\
97.51\end{array}$ \\
\hline $\begin{array}{l}31 \\
33 \\
35 \\
37 \\
39\end{array}$ & $\begin{array}{l}4147 \\
2797 \\
2538 \\
2176 \\
2084\end{array}$ & $\begin{array}{l}0.27 \\
0.18 \\
0.17 \\
0.14 \\
0.14\end{array}$ & $\begin{array}{l}97.78 \\
98.22 \\
98.56 \\
98.86 \\
99.14\end{array}$ & $\begin{array}{l}32 \\
34 \\
36 \\
38 \\
40\end{array}$ & $\begin{array}{l}3808 \\
2658 \\
2516 \\
2091 \\
1569\end{array}$ & $\begin{array}{l}0.25 \\
0.17 \\
0.16 \\
0.14 \\
0.10\end{array}$ & $\begin{array}{l}98.03 \\
98.39 \\
98.72 \\
99.00 \\
99.24\end{array}$ \\
\hline $\begin{array}{l}41 \\
43 \\
45 \\
47 \\
49\end{array}$ & $\begin{array}{r}1538 \\
1301 \\
1117 \\
721 \\
661\end{array}$ & $\begin{array}{l}0.10 \\
0.09 \\
0.07 \\
0.05 \\
0.04\end{array}$ & $\begin{array}{l}99.34 \\
99.52 \\
99.66 \\
99.77 \\
99.85\end{array}$ & $\begin{array}{l}42 \\
44 \\
46 \\
48 \\
50\end{array}$ & $\begin{array}{r}1409 \\
1125 \\
847 \\
666 \\
640\end{array}$ & $\begin{array}{l}0.09 \\
0.07 \\
0.06 \\
0.04 \\
0.04\end{array}$ & $\begin{array}{l}99.43 \\
99.59 \\
99.72 \\
99.81 \\
99.90\end{array}$ \\
\hline $\begin{array}{l}51 \\
53 \\
55\end{array}$ & $\begin{array}{r}598 \\
277 \\
22\end{array}$ & $\begin{array}{l}0.04 \\
0.02 \\
0.00\end{array}$ & $\begin{array}{l}99.94 \\
99.99 \\
100.0\end{array}$ & $\begin{array}{l}52 \\
54 \\
56\end{array}$ & $\begin{array}{r}498 \\
171 \\
15\end{array}$ & $\begin{array}{l}0.03 \\
0.01 \\
0.00\end{array}$ & $\begin{array}{l}99.97 \\
100.0 \\
100.0\end{array}$ \\
\hline
\end{tabular}

Initial and final states for folding analysis of villin headpiece HP35

For calculating the folding pathways we take the following unfolded and folded states, namely ${ }^{3}$

$$
\underbrace{\{3,4,14,21,25,42\}}_{\text {UNFOLDED }} \rightarrow \underbrace{\{1,2,5,6,7,8,12,19,28,40,41,51,55,54,56\}}_{\text {FOLDED }} .
$$


Folding pathways of villin headpiece HP35

TABLE S6: Folding pathways of HP35 (see Eq. (S5)) calculated with MSMPathfinder. A threshold of $P_{\varepsilon}=1 \times 10^{-13}$ was chosen which resulted in an error of $\varepsilon=1.9 \%$.

\begin{tabular}{|c|c|c|}
\hline \# pathway & $P[\%]$ & $P[\%]$ \\
\hline $13 \rightarrow 13$ & 9.57 & 9.57 \\
\hline $23 \rightarrow 9 \rightarrow 1$ & 7.81 & 17.38 \\
\hline $33 \rightarrow 9 \rightarrow 7$ & 3.49 & 20.86 \\
\hline $43 \rightarrow 7$ & 2.76 & 23.62 \\
\hline $54 \rightarrow 16 \rightarrow 6$ & 2.74 & 26.36 \\
\hline $63 \rightarrow 15 \rightarrow 13 \rightarrow 1$ & 2.34 & 28.70 \\
\hline $73 \rightarrow 10 \rightarrow 13 \rightarrow 1$ & 1.44 & 30.14 \\
\hline $83 \rightarrow 1$ & 1.23 & 31.37 \\
\hline $94 \rightarrow 11 \rightarrow 13 \rightarrow 1$ & 1.03 & 32.40 \\
\hline 104 & 0.98 & 33.38 \\
\hline 113 & 0.92 & 34.29 \\
\hline $12 \quad 42$ & 0.87 & 35.17 \\
\hline & 0.61 & 35.78 \\
\hline 144 & 0.61 & 36.39 \\
\hline 153 & 0.61 & 37.00 \\
\hline $164-$ & 0.58 & 37.58 \\
\hline $23 \rightarrow 6$ & 0.55 & 38.13 \\
\hline $184-$ & 0.54 & 38.67 \\
\hline$\rightarrow 16 \rightarrow 6$ & 0.54 & 39.20 \\
\hline 204 & 0.50 & 39.70 \\
\hline $16 \rightarrow 9 \rightarrow 1$ & 0.46 & 40.16 \\
\hline 223 & 0.46 & 40.62 \\
\hline $10 \rightarrow 9 \rightarrow 1$ & 0.46 & 41.08 \\
\hline $17 \rightarrow 13 \rightarrow 1$ & 0.45 & 41.53 \\
\hline $253 \rightarrow 13 \rightarrow 9 \rightarrow 1$ & 0.45 & 41.99 \\
\hline $264 \rightarrow 16 \rightarrow 1$ & 0.44 & 42.43 \\
\hline $273 \rightarrow 16 \rightarrow 6$ & 0.41 & 42.84 \\
\hline $28 \quad 42 \rightarrow 9 \rightarrow 7$ & 0.39 & 43.23 \\
\hline $294 \rightarrow 10 \rightarrow 13 \rightarrow 1$ & 0.38 & 43.61 \\
\hline $30 \stackrel{4}{\rightarrow} \rightarrow 11 \rightarrow 6$ & 0.37 & 43.98 \\
\hline$\rightarrow 13 \rightarrow 1$ & 0.35 & 44.33 \\
\hline $324 \rightarrow 38 \rightarrow 16 \rightarrow 6$ & 0.35 & 44.68 \\
\hline $333 \rightarrow 17 \rightarrow 15 \rightarrow 13 \rightarrow 1$ & 0.33 & 45.01 \\
\hline $34 \quad 3 \rightarrow 24 \rightarrow 7$ & 0.32 & 45.33 \\
\hline $35 \quad 4 \rightarrow 23 \rightarrow 6$ & 0.31 & 45.64 \\
\hline $36 \quad 42 \rightarrow 7$ & 0.31 & 45.95 \\
\hline $37 \quad 42 \rightarrow 8$ & 0.31 & 46.26 \\
\hline $38 \overrightarrow{3} \rightarrow 19$ & 0.31 & 46.56 \\
\hline $393 \rightarrow 8$ & 0.31 & 46.87 \\
\hline $40 \stackrel{4 \rightarrow 7}{\rightarrow}$ & 0.31 & 47.17 \\
\hline
\end{tabular}




\section{Convergence of MSMPathfinder}

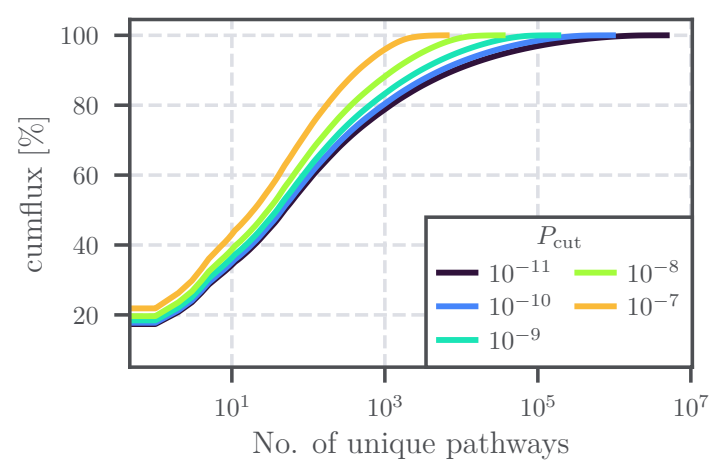

(a) MSMPathfinder

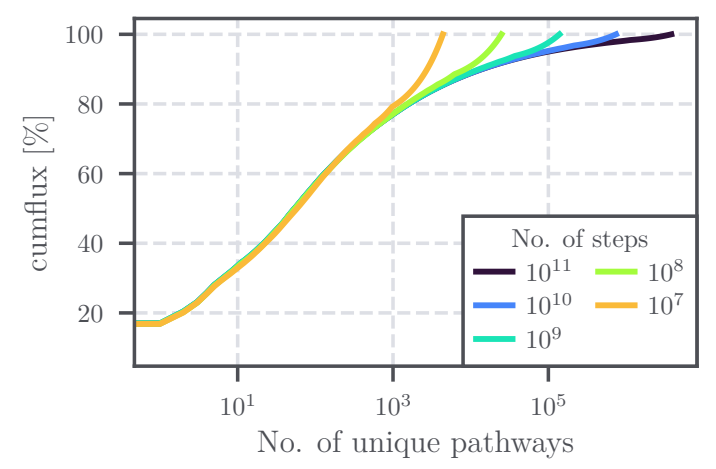

(b) MCMC

FIG. S4: A convergence comparison between (a) MSMPathfinder and (b) MCMC shows the cummulative flux of all pathways sorted by their probability. This reveals that for the first 100 trajectories, MCMC is converging much faster. Nevertheless, this describes roughly about $60 \%$ of the total cumflux. For the remaining part, MSMPathfinder converges much faster.

\section{Waiting times of HP35 folding pathways}

TABLE S7: Mean waiting time when starting in one of the starting states and returning to other starting states is restricted.

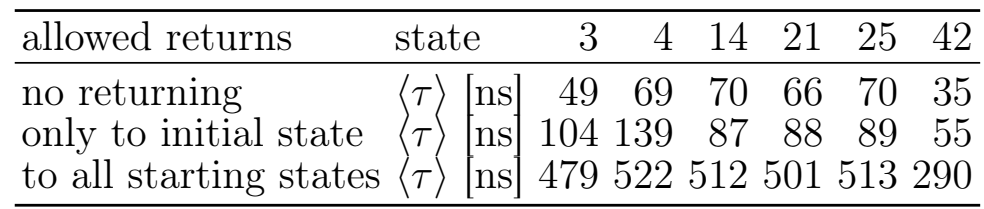

\section{REFERENCES}

${ }^{1}$ D. J. Sharpe and D. J. Wales, Efficient and exact sampling of transition path ensembles on markovian networks, J. Chem. Phys. 153, 024121 (2020).

${ }^{2}$ A. Perez, F. Sittel, G. Stock, and K. Dill, Meld-path efficiently computes conformational transitions, including multiple and diverse paths, J. Chem. Theory Comput. 14, 2109 (2018).

${ }^{3}$ D. Nagel, A. Weber, B. Lickert, and G. Stock, Dynamical coring of Markov state models, J. Chem. Phys. 150, 094111 (2019). 